issue 26: Entanglements - Activish and dech pology

\title{
FCJ-191 Mirroring the Videos of Anonymous: Cloud Activism, Living Networks, and Political Mimesis.
}

Adam Fish

Lancaster University

\begin{abstract}
:
Mirrors describe the multiplication of data across a network. In this article, I examine the politics of mirroring as practiced on videos by the hacktivist network Anonymous. Mirrors are designed to retain visibility on social media platforms and motivate viewers towards activism. They emerge from a particular social structure and propagate a specific symbolic system. Furthermore, mirrors are not exact replicas nor postmodern representations. Rather, mirroring maps a contestation over visibility that entangles both cloud activists and platform firms.
\end{abstract}

doi: $10.15307 / f c j .26 .191 .2015$

\section{Introduction}

In August 2012, Wikileaks was hit with a massive Distributed Denial of Service (DDoS) attack from a mysterious group appropriately titled Antileaks. DDoS assaults occur when multiple 
computers simultaneously 'refresh' a website causing it to overload and shutdown. A shaken-up Wikileaks tweeted: 'The range of IPs used is huge. Whoever is running it controls thousands of machines or is able to simulate them' (Kerr, 2012). This is a constant problem for Wikileaks. Their website reads: 'WikiLeaks is currently under heavy attack. In order to fully protect the CableGate archives, we ask you to mirror it again' (Wikileaks. org). Eventually repelling the Antileaks attack, Wikileaks again took to Twitter to boast: 'DDoS proof, financially \& geographically diverse. We're ready to rumble' (Kerr, 2012). For Wikileaks, mirroring means copying and pasting the CableGate archives in resilient servers so that the content remains visible.

Infamous for Guy Fawkes masks, battles with Scientology and PayPal, support for Wikileaks and Arab Spring activists, hacks of HBGary and Stratfor, braggadocios videos, Lulzsec, and 'Internet freedom,' Anonymous has developed a way for their videos to stay visible on YouTube. The practice they call 'mirroring' is performed when another Anonymous account downloads and then re-uploads the content on YouTube. For the enemies of Anonymous, videos mirrored on different YouTube accounts are difficult to monitor, and if possible, censor. This article is about the politics of data mirrors, what they are and what they do. Below, I introduce mirroring as a political and aesthetic practice similar to but distinct from copying. After detailing my methods which included participating in video mirroring and interviewing Anonymous video producers, I explain what mirrors are, what they are designed to accomplish, and from what social form they emerge.

Mirrors are not merely replicas. They map a contestation over visibility, seek to inspire activism through a process of political mimesis (Gaines, 1999), and evince the complex entanglements of cloud corporations and cloud activists-political actors dependent upon cloud platforms such as corporate social media. I conclude this essay by explicating how the video mirrors of Anonymous emerge from the social form of the living network (Wiedemann, 2014) and are executed to propagate the shared symbolism of an improper name (Deseriis, 2012). In summation, I define mirroring as an emergent practice of a freedom of information movement (Beyer, 2014). As mirroring is an understudied phenomenon practiced across a range of institutions and groups, it was critical to synthesize a wide-range of literature from critical approaches to Anonymous (Deseriis, 2012; Wiedemann, 2014), sociology (Couldry, 2010), feminism (Barad, 2003), and media studies (Askanius, 2013; Gaines, 1999). In the section that follows, I explain the research methods used and provide a brief introduction to Anonymous. 


\section{Methodology and Background}

This article reports on seven years of ongoing research into the video production and dissemination practices of Anonymous. Using YouTube, Twitter, IRC chatrooms, Anonymous websites, and mainstream media, I have tracked Anonymous since 2008 when they first gained public attention because of their offline and online protests against the Church of Scientology. During 2010's Operation Payback, a protest against opponents of piracy and Wikileaks, I began watching hundreds of YouTube videos of all genres, lengths, and technical skill. During their 2011 actions in support of Arab Spring activists, I used my YouTube account to mirror videos produced by Anonymous and thereby gain participatory experiences. As such, this research is an example of politically-engaged 'militant ethnography' in the virtual sphere (Juris, 2007).

By closely following Anonymous on YouTube, I identified the most active producers in terms of output, views, and practical skill. I developed a list of 80 Anonymous YouTube producers to which I sent a questionnaire via YouTube's private user-to-user messaging service. The open-ended questions solicited responses about motivation, inspiration, production practices, and whether or not they mirrored videos. Of the 80 to which I wrote, 14 responded, many in detail. I conducted intensive follow-up interviews with four of these respondents. With the goal of connecting media production practices to reflexive discourse, or what Caldwell (2008) calls, 'industrial reflexivity,' I focus considerable attention on the video oeuvres of interviewees.

Interviewees include THXi330 who has made seven videos that have been viewed over 283,500 times and TheAnonMessage who produced 88 videos with 9,083,428 views. To put this into context, another informant, TheAnonShade, produced 10 videos and slightly more than 2,500 views. Anonymousworldwar3 has made 31 videos with 662,466 views. Alucard 9692010 has made 274 videos with 857,463 views. These are not collective channels but rather single user accounts. The project participants are indicative of the small population of highly competent Anonymous YouTube producers capable of visually evocative and politically aware uses of YouTube. From the differing metrics, it is clear that diverse Anonymous mirror accounts have distinct impacts. Through the course of the research, I encountered YouTube accounts mirroring Anonymous videos along with other less political videos in such a manner that suggests that they were mirroring videos not for the politics but in order to generate advertising revenue. In this article, I focus exclusively on the politically motivated video producers and mirrors, some of whom criticized those mirroring Anonymous videos for profit. Many fractions have emerged within Anonymous with different understandings of what the group is and does. I focus on a small representative of the Anonymous video producers who make and mirror videos on YouTube. 
Since they emerged from the uncensored messageboard 4chan in 2003 , Anons, as they are called, have carried out a range of activities. While not many of those associated with Anonymous are politically motivated, this article focuses on those who participate in Anonymous as a form of political activism. An example of their multifaceted hacktivism was Operation Last Resort in 2013. The operation was initiated to avenge the suicide of freedom of information activist Aaron Swartz (described below), which Anonymous attributed to over-zealous enforcement of outdated computer crime laws. Like all major Anonymous operations, Operation Last Resort was a visual spectacle which included the hijacking of an MIT website to make a Swartz tribute, the production and mirroring of videos, the hacking and releasing of the names and contact information for 4000 banking executives, and the usurping of the U.S. Sentencing Commission website (Blue, 2013). In these actions Anonymous are simultaneously 'part human rights technology activism and part performance spectacle' (Coleman, 2012a: np). While multifaceted, at times paradoxical and always elusive, some insights about Anonymous can be gathered from connecting mirroring practices to social forms and the circulation of symbols. First, however it is important to critically discuss mirrors, what they are, and what they are designed to do.

\section{What Are Mirrors?}

Mirroring is not only for activists. It is also an important practice which enables cloud computing companies to synchronise data in data centres around the world. Microsoft, which provides a number of cloud computing services, defines 'database mirroring' as the maintaining of 'two copies of a single database that must reside on different server instances' (Microsoft, 2014). Microsoft and other companies with immense investments in server farms-Amazon, Google, and Facebook, to name but a few high profile examplesneed to offer robust, secure, and non-delayed access to content. To do this, they strategically locate multiple copies of the same data. Activists also mirror data. Today, sites in eleven European nations offer the Wikileaks mirror (Wikileaks.info, nd). The largest Bittorrent-based peer-to-peer file sharing service in the world, the Pirate Bay, mirrors links to servers located in countries where their service is not yet illegal. Sites like baymirror. com and piratebaymirror.com, enable users to route around legal blockage of root access to the Pirate Bay. For similar goals but on a smaller scale, Anonymous activists download YouTube videos produced by other Anonymous producers and re-upload these same videos on different accounts. Not unlike the mirroring practices of Wikileaks, the Pirate Bay, and Microsoft, this form of mirroring is executed in order to preserve data against politically motivated erasure.

Mirroring has received little focused scholarly attention in critical media studies. Texts like Mirror Worlds (Gelernter, 1992) used the mirror as a metaphor to describe the parallel and 
immersive world that would be created by networked computers. Years before Second Life, and Oculus Rift, Mirror Worlds claimed that virtual worlds were actual places. Today, information companies and information freedom activists alike call data duplication mirroring but often fail to acknowledge how the symbolism of this term may impact its use. Mirrors are more complex entities than simple facsimiles. With multiplication comes the diversification of potential cultural impacts. 'Copying is not merely reproducing the same as discreet objects, but coding cultural products into discreet data and communicating such coded copies across networks: seeding and culturing,' Parikka states (2008: 76). As cultural processes, mirrors echo the intricacies and limitations of data practice. I endeavor to explain how for information activists and information firms, mirroring is an exploit of networks and computers to remain visible through replication.

While mirroring has sustained little attention, copying has received far more, as it relates to major cultural and financial issues such as piracy and precedent setting court cases involving the likes of Napster, Google, Viacom, and the Pirate Bay (Lindgren and Linde, 2012; Postigo, 2012). Copying has largely been framed by academics in terms of a battle between media corporations and the 'free culture' of file sharing. Lessig (2004) has argued that by criminalizing copying, copyright laws dampen cultural innovation surrounding sharing. The political potential of copying can be traced back to the Gutenberg press and the duplication of Lutherian theses, East German facsimiles, and Xeroxes of the Pentagon Papers. Gitelman (2011) emphasizes not the leaking but the photocopying of the Pentagon Papers by whistleblower Daniel Ellsberg as a politicized act of duplication. The unfortunate case of Aaron Swartz, a free culture activist caught downloading thousands of academic journal articles, which he intended to redistribute through mirrored databases, who took his own life in 2013 amidst a prosecution, speaks to the fervor surrounding today's copy culture.

The politics of copying brings us to the early days of free and open source software (FOSS). Millions of computer operating systems run on FOSS such as GNU/Linux which was created by volunteer coders copying and sharing source code (Kelty, 2008). For the FOSS community, copying is a technical practice, an instigator for community, and a moral imperative (Coleman, 2012b). Anonymous and the Pirate Bay share with the FOSS community a belief that copying is practically necessary, socially integral, and an inalienable right (Halliday and Arthur, 2010). Co-founder of the Pirate Bay, Peter Sunde, believes that we are genetically coded to copy: 'People learn by copying others. All the knowledge we have today, and all success is based on this simple fact - we are copies' (cited in Ernesto, 2014). For the Pirate Bay, mirroring is necessary to secure the visibility and accessibility of their file-sharing service. Video mirroring is an activist labor that seeks to overcome or bypass copyright regimes and censorship. Beyer (2014) sees in Anonymous, Wikileaks, and the Pirate Party an emergent 'freedom of information movement.' Mirroring is a little understood practice of this movement. 
Video mirroring is both practical and aesthetic. From the Dada cut-up techniques of the 1920 s to punk rockers in the 1970s resignifying the safety pin, copying, pasting, and recontextualizing have long been important sources of aesthetic creativity. Today's video activist is also a bricoleur who usurps and remixes found sound and footage. Manovich (2001) makes clear how the aesthetics of software recontextualize the past. For example, Navas (2010) describes Apple's original graphic user interface as a copy of the IBM command line remixed with the metaphor of a 'desktop.' He describes 'regenerative remixes' (Navas, 2010) which are social media sites like Tumblr that use interoperable APIs to copy and remix content into ever-changing, real-time, personalized sites. Like regenerative remixes, mirrors result from the copy/paste functionality of computers, networks, and social media platforms. Unlike video and API mashups, however, mirrors are icons that are aesthetically similar to source material instead of being unique iconoclasms.

The political power of mirrors is achieved through bestowal, homogeneity, and ubiquity while the politics of remix is achieved through appropriation, heterogeneity, and novelty. Mirrors are centrifugal, their constitution requires 'seeding' (Parikka, 2008: 76). Remix is centripetal, produced through a mode of gathering (McKelvey, 2014). Video mirrors of Anonymous are different from peer-to-peer file sharing for the simple reason that the shared files reside in proprietary cloud servers and not on private personal hard drives. The sharing of video mirrors happen within a single cloud system while peer-to-peer sharing transects proprietary, public, and private platforms. In this manner, Anonymous video producers are dependent upon corporate cloud platforms such as YouTube and Google.

A common belief is that mirrors are exact replications, merely displaced within databases. A more complex social constructivist perspective sees mirrors as symbolic representations. Barad (2003) challenges both 'naïve realist' as well as constructivist interpretations of scientific knowledge. She offers a third interpretation of mirrors when she says '...the representationalist belief in the power of words to mirror preexisting phenomena is the metaphysical substrate that supports social constructivist, as well as traditional realist, beliefs' (Barad, 2003: 802). Mirrors are neither realist copies nor constructed depictions. They offer viewers another perspective on a practice of visual contestation. Mirrors are performances enacted within and between relational databases with the goal of securing video power.

Mirrors can be read as constitutive material practices that point to frictions and paradoxes of what can be called cloud activism. This interpretation of mirrors requires a shift from reflection to diffraction that "does not produce "the same" displaced, as reflection and refraction do. Diffraction is a mapping of interference, not of replication, reflection, or 
reproduction. A diffraction pattern does not map where differences appear but rather maps where the effects of differences appear' (Haraway, 1992: 300). Anonymous video mirrors are diffractions that visually map a contestation over networked visibility.

Video mirroring is but one practice of visibility indigenous to a particular class of cloud activism. This is a preliminary attempt at defining one small iteration of mirroring that may link in practice to other forms of politicized copying exercised by the freedom of information movement (Coleman, 2014; Beyer, 2014). Below, I critically analyse mirrors, what they do, as well as the social formations from which they come.

\section{What Do Mirrors Do?}

The mirrors of Anonymous attempt to do two things: 1) proliferate and preserve videos so that they might 2 ) motivate others to mimic the radical personae represented in the videos.

\section{Mirrors as a Will to Visibility}

Video mirroring can be theorised in terms of a will to visibility. The term 'will to visibility' refers to both the desire and the capacity to be seen. It denotes the 'intrinsic, rational, non-empirical ground of social process' that accounts for both the 'strategies' as well as the 'conditions of visibility' (Criado, 1995: 197). Mirrors map a will to visibility not unlike the act of voicing. Couldry (2010: 7-10) defines the act of giving voice as a process that is socially grounded, reflexive, agential, embodied, material, and valuable beyond market fundamentalism. Anonymous videos are a visualisation of voice. This will to visibility is contested by the 'scopic regimes' of Western technoscience and statecraft that reserve the power to make something visible, legible, and able to be controlled (Haraway, 1997; Jay, 1992; Scott, 1999). Historically, writing and printing prioritised the ocular and thus gave power to those who could read, write, and print, and therefore cast mortal judgments based on ledger knowledge (McLuhan, 1964; Ong, 1977). Thus, visibility 'lies at the intersection of the two domains of aesthetics (relations of perception) and politics (relations of power)' (Brighenti, 2007: 324). In short, power is enacted through the capacity to make things visible. Mirroring retains visibility for activist video while also making evident the entanglements of cloud activists and information corporations. Mirrors represent a proliferation of content to avoid erasure, maximising the potential for political recruitment. 
Anonymous as a political project began in 2008 when a video was leaked on YouTube of Tom Cruise energetically celebrating Scientology. Scientology initiated a comprehensive campaign to have the video deleted from YouTube. Lawyers for Scientology claimed the video infringed upon their copyright (Denton, 2008). According to my informants, Scientologists were instructed to 'flag' this video as inappropriate before it was rapidly mirrored across YouTube. Flagging refers to the option provided by YouTube to users to notify YouTube that they consider the content offensive or copyrighted. 'Flags,' according to Crawford and Gillespie (2014: 3), 'act as a mechanism to elicit and distribute user labor-users as a volunteer corps of regulators.' Reducing online commentary to simplistic binaries of "offensive" versus "inoffensive," flags potentially diminish the generative potential of agonistic debate over what has the right to remain visible. An unknown quantity or frequency of flags and the video is automatically removed until the user petitions to have it reinstated. To avoid this, dedicated mirror channels exist to insure that flagging actions are distributed across a number of mirrors making it difficult to censor all Anonymous videos. This was the beginning of the Anonymous war against Scientology, Project Chanology, which was announced through a heavily mirrored YouTube video, Message to Scientology (ChurchOfScientology, 2008). In this and subsequent operations, video mirroring played a crucial role in Anonymous securing visibility. Mirroring is a diffraction; it creates a militant topology, or a mapping of conflict.

A second video followed from YouTube user ChurchOfScientology, Call to Action, sought to combat mainstream media misrepresentation of Anonymous as 'superhackers' and not a 'collective...from all walks of life' (ChurchOfScientology, 2008). Call to Action concluded by inviting viewers to protest outside of Scientology centres on February 10th, 2008. Because of what some Anons consider to be another politically motivated flagging initiative, Call to Action was taken down by YouTube, which cited a violation of 'terms of service.' Soon after, however, the video was mirrored across YouTube. In operations linked to the video, Anonymous began DDoS confrontations, prank calling, and sending black faxes and unpaid pizza to Scientology headquarters. In these ways, video was the means for political mobilisation. The impact of the videos would have been minimised were it not for mirrors.

Code of Conduct (ChurchOfScientology, 2008) was a third video of Project Chanology. Where Message to Scientology was addressed to Scientology and Call to Action to the mainstream media, Code of Conduct was a public document for other Anons citing 22 principles to follow for successful physical protests. Code of Conduct was vigorously mirrored. Anonymousworldwar3 explained to me why they mirrored Code of Conduct: 'at that time we were organising real life protests and I felt it was important to get that video/ info out there before going out into the "real world". Despite the flagging that Anonymous assumed was resulting from Scientology, all three of these videos remained visible because of the practice of mirroring. 
According to user AnonymousFacts, Scientology forced YouTube to delete the account and several others that were instrumental in making and releasing these anti-Scientology videos (AnonymousFacts). In the video, A Call for Video Mirroring, Anonymousworldwar3 requested viewers to download and re-upload their Arab Spring videos. The narration states:

I noticed someone started flagging these video's as inappropriate and/or voting them down systematically. Apparently, not all agree to the right of free speech... As much as I regret this, there is nothing I can do to prevent this. That is why I want to ask all of you to mirror these video's before it is too late (Anonymousworldwar3, 2010).

In the comments, user @CavalierPosts asks how to mirror and Anonymousworldwar3 responds with instructions. For Anonymousworldwar3 being a centralised hub for videos made Anonymous vulnerable while decentralisation through mirroring distributed risks of visual erasure across the network. Dependent as cloud activists are on corporate platforms, the highly mobile practice of mirroring, which distributed risk across the platform, made Anonymous less vulnerable. Mirroring is a way Anonymous resisted Scientology's counterattack. Interviewee Alucard 9692010 agreed:

Back in 2008 when Anonymous started its war against the Cult of Scientology, a very large quantity of Anonymous videos were created to educate and expose Scientology to the general public. But then I (and many others) noticed that Scientology started a false flagging campaign and took down quite a few of these anonymous videos. So, as a response, Anonymous everywhere (me included) started to re-upload the removed videos everywhere. Nobody likes a cheater :). They took one of our videos down, we uploaded it 10 times again on 10 different channels. Even people that had nothing to do with Anonymous started to upload our videos on their channels when they noticed the Scientology false flagging campaign. This is how this channel has started. Since then it evolved, uploading mirrors of videos in chronological order following certain stories as they happened (ACTA, occupy movement, the Arab spring and so on). A anonymous information channel.

Both Scientology with their alleged flagging campaign and Anonymous with their mirroring practices, exploited the affordances provided by YouTube. Ostensibly a way for YouTube users to help govern the site (Crawford and Gillespie, 2014), flagging is inherently political. The political application of mirroring too evolved out of YouTube's desire for scale and growth by not limiting user-account registrations to specific Internet protocol addresses. 
In this way, both Scientology and Anonymous exploited an affordance as a weapon in a semiotic war. As I will describe in full below, this entanglement is indicative of cloud activism.

Video mirroring is a practice that exploits the scalability of social media platforms to maximise visibility and resist erasure through flagging. Unlike video production, mirroring does not require cinematographic or editing skills but rather competencies in downloading, uploading, and user account management. For example, TheAnonShade focuses exclusively on mirroring videos about Anonymous operations in the Middle East and Asia. They describe their activities:

I am a messenger of the Shadowy part of Anonymous, I just mirror videos that NEED and have the itching to get out. My role is the other side of the world; which is Syria, Bahrain, India, and China. ... I, as well as my team have access to one of the biggest Anons. So when they are about to upload a video immensely important to the collective. We are the first people to do a first reupload to their specific video.

As mirroring is a mapping of a displacement so too are mirroring actors geographically dispersed. Anonymous videos are incorporated into operations against the politically powerful such as Syrian President Bashar Al-Assad and organisations such as Scientology, the NSA, and PayPal with the economic resources to retaliate against antagonists. Mirrors utilise the replicability of digital information, the distributed nature of networks, and the pseudo-anonymity provided by some social media platforms to create an innovative form of visibility. As THXi330 said, mirrors enable Anonymous to explore the "possibilities of viralisation.' Mirroring is one experiment with viralisation that exploits pseudo-anonymity, replicability, and networks. Mirroring is both an offensive manoeuvre to propagate symbols and circulate affect and a defensive manoeuvre to remain visible in a contested and privatised cloud-based public sphere. Remaining visible is the goal on the infrastructural level while political mimesis, the hailing of bodies to feel and move like the revolutionary beings depicted on video screens, is the aim on the subjective level.

\section{Mirrors as Political Mimesis}

Because of their verisimilitude, mirrors are central metaphors with which to frame representation, identity, and symbolism. One contribution of postmodernism is 
constructionism. A reaction to realism, constructionism is relativistic in its interpretation of representations. Taussig (1980) interprets rituals as the personification of icons and as invitations for others to embody the personified icons. These mimetic rituals are often augmented by masks that temporarily transform initiates into liminal states of shared pseudonymity. Gaines (1999) blends the work of Taussig and Williams (1991) to develop a theory of 'political mimesis,' or how documentaries seek to move the bodies of audiences. Drawing from Williams (1991) and her typology of bodily reactions to horror (scream), melodrama (cry), and pornography ('come'), Gaines (1999) describes a fourth genre, the political documentary, which is designed to motivate viewers to take up political action. Gaines (1999: 93) differentiates mimesis from mimicry that carries 'connotations of naïve realism, mindless imitation, mechanical copying, and even animality'. Anonymous represents a politically engaged, networked form of video production and masked activism that calls upon supporters to mirror videos and copy revolutionary bodies. This type of mimesis is not "naïve realism," rather it is a form of corporeality more immediate than second-order representation. The Guy Fawkes mask enables participants to mimetically embody a radical affect. It provides high visibility and shared symbolism while retaining pseudonymity on corporate social media.

Anonymous YouTube producers are examples of what Anonymous calls 'moral fags.' With their emergence from the shady underworld of the imageboard 4chan, Anonymous uses this crude language to describe politically active Anons as opposed to those who are just in it for fun, trolling, or the 'lulz'. Moral fags produce politically motivated videos that attempt to 'pump up' supporters and bring 'melancholy' to their enemies as THXi330 and TheAnonMessage informed me. One informant, Anon Pyrriel, self-identified as a moral fag:

In other words I am with the part of Anonymous that fights for all that is unjust in our opinion. I am not affiliated with the part of Anonymous that posts flashing pictures on epilepsy websites and tries to 'troll' the Internet.

Worldunitedanonymous explains his motivation for posting political videos in this way:

Videos calling for peace and justice impact me the most. For example during Oplsrael several videos surfaced calling for peace in the region, even when both sides had things to be angry at the other about. I find stuff like that really inspirational because it shows that humanity can come together in the name of peace even when no sign of peace is in sight. 
Anonymousworldwar3 uses 'imagery of protest because I believe these images have the ability to inspire people to get out there and protest themselves, while it also gives the videos a certain epic quality and stimulates a feeling of agency in the viewer.' Worldunitedanonymous reiterated that 'moral fags' produce videos to bring about world peace and end greed, corruption, and exploitation. Alucard 9692010 said 'moral fags' agitate to defend freedom of information, free speech, and an unregulated Internet. These videos can be seen as 'radical media' (Downing, 2001) or 'mobilization videos' (Askanius, 2013)-forms of communicative action that attempt to galvanise support for political operations.

Downing (2001) describes radical media as content that invites audiences to participate in social movements and media production. Radical media enters into paradoxical relationships with corporately owned Internet platforms such as YouTube. In the case of Anonymous, 'radical' is a preferable term to 'alternative,' 'participatory,' or 'independent' which are either hegemonic in their complicity with dominant media systems and corporations or counter-hegemonic in their attempts to reform hegemonic systems. Radical media is revolutionary and anti-hegemonic, seeking transformation of the present system. Anonymous is a radical movement in its no compromise rejection of states' rights to surveillance and their extreme views on free speech maximalism and 'radical transparency' (Sifry, 2011). Yet while their perspective on the Internet as an information commons can be decisively anti-corporate and post-capitalist, the paradox is that radical media often use corporate and increasingly centralised media platforms such as YouTube to perform their radical will to visibility.

According to DCHTID247, the goal of political mimesis is 'to mirror to the world that there is non-hierarchical groups out there that are fighting for freedom.' Anonymousworldwar3 maintained: '...anon is just a symptom of this new structure imposing itself on today's world: it is one of the first glimpses of a "new world" that unfolds itself on the basis of this new communication and information-infrastructure and the principles that organise this.' In this way, Anonymous videos are 'mobilisation videos' which do not seek to re-affirm social movement solidarity but rather seek to galvanise adherents in a particular cause (Gregory et al., 2005). Because they attempt to energise those already converted, mobilisation videos tend to be reductive and fundamentalistic. Askanius (2013) categorises political mash-up videos as examples of mobilisation videos with three unique qualities: aesthetic generativity, genre hybridity, and production democratisation. Anonymous videos are an example of the 'coalition model' of radical media wherein the goal is not a finalised film or fame for the producer, but rather the initiation of a political process (Christiansen, 2009). 
For those willing to talk, either rationally or passionately, the Internet can constitute the conditions for a public sphere (Habermas, 1987), a digital public sphere (Dahlgren, 2005), a subaltern counterpublic (Fraser, 1990), or an agonistic and passionate public sphere (Mouffe, 2005). While YouTube has proven to be an important site for political debates, mobilisation videos like those made by Anonymous are clearly not designed to simulate a version of a public sphere. In the case of Anonymous, viewers leave comments but producers rarely comment back to viewers. The majority of the commentary on Anonymous videos consists of declarations of support or troll-like denouncements. This is video as a direct will to visibility. It is propaganda to mobilise supporters, not media to provoke reasoned, agonistic, or subaltern debate. In short, Anonymous videos magnify a collective affect and YouTube is the platform for this shared feeling.

On one level, Anonymous mirrors are representations. They are an invitation to viewers to enact political mimesis by modeling themselves after masked revolutionary bodies depicted on screen. On another level, by not pointing to an ontogenetic moment but rather being an ongoing struggle, Anonymous mirrors are a form of praxis transfigured by conflict, affectually shared, and existing in a paradoxical relationship to information firms. Either as political mimesis or practice, Anonymous videos represent sophisticated adaptations to network affordances in a pursuit of visibility.

Mirroring is a practice that emerges from a particular social structure designed to propagate a specific set of symbols. The terms 'cloud activism,' 'living networks,' and 'improper names' may assist us in understanding how mirrors emerge from the structural and symbolic systems of Anonymous

\section{Structures and Symbols of Anonymous}

Anonymous is an example of 'cloud activism' entangled in paradoxical relationships with for-profit social media firms (Milan, 2013; Nielsen, 2009). Bringing to the theory of cloud activism the concept of the 'living network' adds a missing dimension of the importance of affect in the formulation of Anonymous (Stoehrel and Lindgren, 2014; Thacker, 2004; Wiedemann, 2014). I add the notion of collective pseudonyms or 'improper names' in order to discuss the important role played by symbols in the growth of Anonymous (Deseriis, 2013). Mirroring is an activist practice particular to a structure of feeling and symbolism requiring the synthesis of these theories to explain. 


\section{Cloud Activism}

Utilising geographically dispersed servers, cloud computing allows users to access remote data and applications. 'Cloud activism' is 'activism based on a structure that the user does not control or own the way they would an individual centralised personal computer' (Nielsen, 2009: 279). Cloud activism is activism that is dependent on privatised information infrastructure. As a form of activism performed on freely accessible and privately held social media platforms such as YouTube, mirroring is an example of cloud activism entwined with servers and databases held in private.

Based on her ethnographic research into the relationships between environmentalists, indigenous people, and illegal loggers in the rainforests of Indonesia, cultural anthropologist Anna Tsing identified that the places these actors meet constitute a 'zone of awkward engagement' that is characterised by friction or the 'awkward, unequal, unstable, and creative qualities of interconnections across difference' (Tsing, 2005: 4). These interconnections 'remind us that heterogeneous and unequal encounters can lead to new arrangements of culture and power' (Tsing, 2005: 6). For Anonymous, YouTube is a 'zone of awkward engagement' wherein their competencies with software, social media, and video permit a small and awkward shifting of video power. The mirroring practices of Anonymous are a form of cloud activism that exposes the imbroglio of information activists using profitseeking social media platforms. As Gillespie (2010) articulated, the semiotics of 'platforms' is intentionally left open so as to remain versatile. Through mirroring, Anonymous exploits not just the semiotic but also the practical variability of social media platforms.

User Anonymousworldwar3 describes its entanglement with cloud-based social media firm YouTube:

At a certain point in time, this was (Anonymousworldwar3) the largest anon channel on YouTube, which I think was not a great thing for a number of reasons: it made anon's presence on youtube quite vulnerable, since it allowed people to take just one channel down and most of anon would be wiped from youtube + it gave me a far too central role within anon, which goes against the concept of decentralization within anon itself. So we started to make sure all of the anon videos were mirrored on a number of youtube channels + where at first we were only with two or three people doing videos, we started some sort of 'video-training' to make sure there would be a whole lot more people making videos, both for the sake of being able to hide within a larger 
crowd, and to make anon's youtube presence less centralized and thus less vulnerable.'

For Anonymous and their targets such as the U.S. Sentencing Commission in 2013's Operation Last Resort, YouTube constitutes a 'zone of awkward engagement.' The asymmetries in this interdependence may result in a temporary shifting of the power of visibility. This visibility is partly enabled by knowing how the platform works: it's rules, expectations, and weaknesses. Anonymous is a living network that understands how to (mis)use cloud-based social media platforms to generate and circulate affect.

\section{Living Networks}

While describing the relation of Anonymous to proprietary social media platforms, cloud activism does not explain the affect that links activists in circulations. Gregg and Seigworth (2010: 1) define affect as the visceral forces that 'drive us toward movement, toward thought and extension.' Hansen (2004) considers affect a key aspect in the process of engagement with digital information (see also Featherstone, 2010). Similarly, Lazzarato treats digital technologies as 'motors of affective energy' (2007: 115). It is the combined emotional intensity and behaviour that binds individuals within Anonymous, not collective 'identities, myths, or narratives' (Wiedemann, 2014: 6). Anonymous is a 'living network' that is 'inherently dynamic, undergoing constant and variable changes, both within the composition of individual nodes, and in the relations between nodes' (Thacker, 2004: np). In emphasising movement and historical change and de-emphasising static nodes, Thacker underscores not 'network effects' but 'network affects' (Thacker, 2004: np). As Stroehel and Lindgren (2014) identify, the affective intensities of Anonymous drive their political projects. Anonymous as a variegated 'living network' can be observed not only through mirroring but also through their DDoS assaults.

Mirroring, along with DDoSing, is a tactic that one does not need to be a hacker to do. While the United States courts have yet to protect it as a form of political speech, some activists consider DDoS a valid form of 'electronic civil disobedience' (Critical Art Ensemble, 1996). In 2012, Anonymous successfully DDoSed a tear-gas manufacturer in Pennsylvania, the Nasdaq stock exchange, the U.S. Department of Justice, the Federal Bureau of Investigation, the Central Intelligence Agency, the White House, Amazon, eBay, CNN, Buy.com, Paypal and Yahoo, and websites of the Tunisian and Syrian governments (Sauter, 2013). Anonymous used a special tool to successfully DDoS enemies. The low-orbit ion cannon (LOIC) is simple software that sends repeated website refresh requests. It 
'opened the tools and mechanisms of protest organizing and action to the population of the Internet' (Sauter, 2013: 2). Like mirroring, with LOIC anyone can participate.

One feature of LOIC, was a button titled 'fucking hive mind mode' that allowed users to let their computer be controlled by a remote computer. This software gives us insights into the structure of the living network. As Coleman (2012a: np) states, Anonymous embodies 'meritocratic populism' while also resisting 'possessive individualism'. Mirroring is not static but a practice of 'rhizomatic nomadic warfare' (as one of my informants, THXi330, said paraphrasing Deleuze and Guattari 1987) with sudden, ephemeral, and nodal emergences. LOIC, and the 'hive mind' functionality in particular, make evident how Anonymous functions as a rhizomatic living network constituted not by nodes but by edges and the "motors of affective energy" (Lazzarato, 2007: 115). Likewise, mirroring is a practice of a living network alloyed by affect, distinguished by movement across a topology. While 'living network' is a useful heuristic with which to understand the social form of Anonymous it does not account for the importance of shared symbolism.

\section{Improper Names}

Anonymous is not, actually, anonymous. It is pseudonymous. Like the Luddites taking on the name of fictional character Ned Ludd, Anonymous is an 'improper name' (Deseriis, 2013: 35), a shared pseudonym with common symbolic traits. 'Anonymous', is a symbolic system, that enables participants to recognise each other and permits a process of 'subjectivation' (Deseriis, 2013: 35). The concept of subjectivation, or the construction of subjectivity, is key to understanding the unitary power of the improper name and requires an understanding of the term 'dividual' (Deleuze, 1992). Social media firms decompose individuals into separate 'dividual' transactions, metrics, preference, and discreet acts (Deseriis, 2013: 35). An inclusive living network with an improper name such as Anonymous, 'resists capital's attempts to break down the continuity of social praxis in dividual and discrete units' (Deseriis, 2013: 46). The resistance transforms dividuals into collective codividuals. The shared visual and aural symbolism surrounding the improper name Anonymous-most notably the Guy Fawkes mask and video aesthetics-constitutes the codividual symbolism of this living network. Mirroring is a practice that propagates this symbol.

Anonymous has an extraordinarily rich symbolic culture. For an 'improper name' with no official spokesperson, their graphic expressivity provides some of the densest displays of their values. As Coleman (2010: $\mathrm{np}$ ) asserts, '[i]ts aesthetic also helps ensure some 
coherence....while anyone can claim to be Anonymous, you will likely [be] more credible if you follow and play with established patterns.' The smiling black-on-white Guy Fawkes mask popularised in the 2005 movie $V$ for Vendetta is their most prominent symbol representing anonymity, playfulness, and resistance. Distinguishing characteristics of the shared symbolism of Anonymous videos include masked figures, the introductory logo, the text-to-speech (TTS) narration, intense classical or techno music, and the traditional concluding remarks 'we don't forgive, we don't forget, expect us.' Some videos have the quality of masked newscasters, others of hostage videos. The writing is always pedantic and serious, using either first person (us) or second person (you) modes of address. Other than the necessary YouTube account pseudonym, never are authors cited.

Most videos are quite simple, featuring the opening circular and rotating Anonymous logo, a masked figure gesturing, and a TTS intoned declaration. Some are very ornate requiring highly skilled editing. Three of these elements, the logo, icon, and the TTS tonality, are transposable and transferable assets capable of being appropriated to speak any declaration. Anyone can download and edit together the globe spinning preroll, a Philip Glass soundtrack, a gesticulating character in a Guy Fawkes mask, a TTS voice program, an apocalyptic text-and produce an Anonymous video. These foreboding visuals, robotic sounds, and ominous narratives form much of the shared symbolism of Anonymous as an 'improper name.'

World United Anonymous describes Anonymous as an improper name when he directly addressed me, saying:

By default you are already Anonymous, Anonymous in general is an idea, as said before, everyone is entitled to an idea. If you choose to use the name, you can help by spreading knowledge, making videos, music, tweeting, just being a good person, etc.... The name 'Anonymous' just helps put all our ideas in one place so people find them easily. Anonymous is not a group, nor is it an army, person, or even a movement. Anonymous is simply a main idea which is home to a collective of ideas.

'Anonymous' is a pseudonym anyone can adopt that provides the conditions for 'generativity, democracy, and instability' (Coleman, 2010: np). Mirroring is an act of visual resistance that distributes this symbolic system against the threat of deletion.

As a form of cloud activism, Anonymous is entangled with the platform-owning corporations 
they resist. As a living network, they have a fluctuating topology formed by the circulation of affect. The improper name 'Anonymous' is constituted by shared symbols. Like DDoSing, mirroring is a cultural practice specific to this formulation of radical media paradoxically dependent as it is on cloud infrastructure, the circulation of affect, the hope of political mimesis, and a shared pseudonymous symbolism. Mirrors are not mere representations. They mark a will to visibility and chart a terrain of diffraction.

\section{Conclusion}

Caught once again looking at mirrors, it is either the face of transcendence or our own image. It is as if there are no alternative ways to conceptualize matter: the only options seem to be the naiveté of empiricism or the same old narcissistic bedtime stories (Barad, 2003: 827).

Cloud firms may assume the 'naïvete of empiricism' (Barad, 2003: 827) or 'naïve realism' (Gaines, 1999: 93) where database mirroring is confidently practiced and asserted to gain investor and client trust that files will not be deleted or slow in opening. In this case, mirroring is logically positive, an instrumental practice of duplication, and a form of mimicry not mimesis. Barad calls for ways of discussing the world without simplistic reductions to mimicry. We need neither to empirically conceptualise mirrors as accurate copies nor must we consider them as constructed reflections. This article has considered a third way of interrogating mirroring within cloud activism by a living network with an improper name. Mirroring is a praxis of a living network, neither realistic nor representational, but a way of sharing affect, propagating collective symbols on cloud platforms, and hailing codividuals towards political mimesis. Anonymous uses the affordances provided by social media firms to challenge scopic regimes (Jay, 1992) that would silence it. This will to visibility is shared by a number of high-profile cloud activists. Along with the Pirate Bay, the Pirate Party, and Wikileaks, Anonymous may form a 'freedom of information movement' (Beyer, 2014). Mirroring, as an adaptive way of resisting erasure while exploiting the properties of proprietary social media, may become a key practice of this new social movement.

\section{Author Biography}

Adam Fish is a social anthropologist of digital culture, business, and politics at Lancaster University. He investigates the interface of economic and political power, cultural 
discourses and practices, and networked communication technologies. These interests coalesce into critical and ethnographic investigations into media industries and media activism.

\section{References}

AnonymousFacts. YouTube (nd) https://www.youtube.com/user/AnonymousFacts

Anonymousworldwar3. 'ANONYMOUS - A Call For Video Mirroring', YouTube (2010), https:// www.youtube.com/watch?v=MBkGvPTDryw

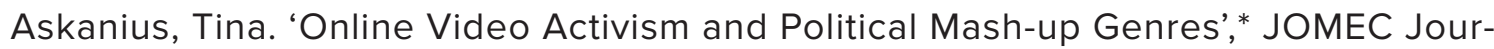
nal Journalism Media and Cultural Studies *(2013) https://lup.lub.lu.se/search/publication/4180637

Barad, Karen. 'Posthumanist Performativity: Toward an Understanding of How Matter Comes to Matter', Signs: Journal of Women in Culture and Society 28.3 (2003): 801-831.

Beyer, Jessica. Expect Us: Online Communities and Political Mobilization (Oxford: Oxford University Press, 2014).

Blue, Violet. 'Anonymous Hacks US Sentencing Commission, Distributes Files', ZDnet (2013) http://www.zdnet.com/article/anonymous-hacks-us-sentencing-commission-distributes-files/

Brighenti, Andrea. 'Visibility: A Category for the Social Sciences', Current Sociology 55.3 (2007): 323-342.

Caldwell, John. Production Culture: Industrial Reflexivity and Critical Practice in Film and Television (Durham: Duke University Press, 2008).

Christiansen, Christian. 'Political Documentary, Online Organization, and Activist Synergies', Studies in Documentary Film 3.2 (2009): 77-94.

ChurchOfScientology. 'Message to Scientology', YouTube (2008) https://www.youtube.com/ watch?v=JCbKv9yiLiQ

Coleman, Gabriella. 'The Aesthetic Face(s) of Anonymous', Savage Minds (2010) http://savageminds.org/2010/12/15/aesthetic-face-of-anonymou/

Coleman, Gabriella. 'Our Weirdness Is Free, The logic of Anonymous-Online Army, Agent of Chaos, and Seeker of Justice', Triple Canopy (January 2012a), http://canopycanopycanopy.com/issues/15/contents/our_weirdness_is_free

Coleman, Gabriella. Coding Freedom: The Ethics and Aesthetics of Hacking (Princeton, N.J.: Princeton University Press, 2012b).

Coleman, Gabriella. Hacker, Hoaxer, Whistleblower, Spy: The Many Faces of Anonymous 
(London: Verso, 2014).

Couldry, Nick. Why Voice Matters (London: Sage, 2010).

Crawford, Kate and Tarleton Gillespie. 'What is a Flag For? Social Media Reporting Tools and the Vocabulary of Complaint', New Media and Society (2014).

Criado, Felipe. 'The Visibility of the Archaeological Record and the Interpretation of Social Reality', in Ian Hodder, Michael Shanks, Alexandra Alexandri, Victor Buchli, John Corman, Jonathan Last, and Gavin Lucas (eds). Interpreting Archaeology: Finding Meaning in the Past (New York: Routledge, 1995).

Critical Art Ensemble. Electronic Civil Disobedience and Other Unpopular Ideas (New York: Autonomedia, 1996).

Dahlgren, Peter. 'The Internet, Public Spheres, and Political Communication: Dispersions and Deliberation', Political Communication 22.2 (2005): 147-162.

Deleuze, Gilles. 'Postscript on the Societies of Control', October 59 (1992): 3-7.

Deleuze, Gilles and Felix Guattari. A Thousand Plateaus: Capitalism and Schizophrenia (Minneapolis: University of Minnesota Press, 1987).

Denton, Nick. 'Church of Scientology Claims Copyright Infringement', Gawker (2008) http:// gawker.com/5002319/church-of-scientology-claims-copyright-infringement

Deseriis, Marco. 'Improper Names: Collective Pseudonyms and Multiple-Use Names as Minor Processes of Subjectivation', Subjectivity 5.1 (July 2012): 140-160.

Downing, John. Radical Media (London: Sage, 2001).

Ernesto. 'Pirate Bay Founder Gets Ready to Run for European Parliament', Torrentfreak (2014), https://torrentfreak.com/pirate-bay-founder-peter-sunde-arrested-sweden-140531/

Featherstone, Mike. 'Body, Image and Affect in Consumer Culture', Body \& Society 16.1 (2010): 193-221.

Fraser, Nancy. 'Rethinking the Public Sphere: A Contribution to the Critique of Actually Existing Democracy', Social Text 25.26 (1990): 56-80.

Gaines, Jane M. 'Political Mimesis', in Jane M. Gaines and Michael Renov (eds). Collecting Visible Evidence (Minneapolis: University of Minnesota Press, 1994). Gelernter, David. Mirror Worlds, or: The Day Software Puts the Universe in a Shoebox... How It Will Happen and What It Will Mean (Oxford: Oxford University Press, 1992).

Gillespie, Tarleton. 'The Politics of 'Platforms', New Media and Society 12.3 (2010): 347364.

Gitelman, Lisa, 2011. 'Danial Ellsberg and the Lost Idea of the Photocopy' in Andrew Ekstrom, Solveig Julich, Frans Lundgren, and Per Wisselgram (eds). History of Participatory Media, Politics and Publics 1750-2000 (New York: Routledge, 2011), 112-124. 
Gregg, Melissa and Gregory J. Seigworth. 'An Inventory of Shimmers', in Melissa Gregg and Gregory J. Seigworth (eds). The Affect Theory Reader (Durham, NC: Duke University Press 2010), 1-25.

Gregory, Sam, Gillian Caldwell, Ronit Avni, Thomas Harding. Video for Change: A Guide for Advocacy and Activism (London: Pluto, 2005).

Habermas, Jurgen. The Theory of Communicative Action (Boston: Beacon Press, 1987).

Halliday, Josh and Charles Arthur. 'Wikileaks: Who Are the Hackers Behind Operation Payback?', the Guardian newspaper (2010) http://www.theguardian.com/media/2010/dec/08/ anonymous-4chan-wikileaks-mastercard-paypal

Hansen, Mark B.N. New Philosophy for New Media (Cambridge, Mass.: MIT Press, 2004).

Haraway, Donna. 'The Promises of Monsters: A Regenerative Politics of Inappropriate/d Others in Cultural Studies', in Lawrence Grossberg, Cary Nelson, Paula A Trechiler (eds). Cultural Studies (New York: Routledge, 1992).

Haraway, Donna. Modest_Witness@Second_Millennium.FemaleMan@Meets_OncoMouse ${ }^{\mathrm{T}}$ : Feminism and Technoscience (New York: Routledge, 1997).

Jay, Martin. 'Scopic Regimes of Modernity' in Hal Foster (ed.). Vision and Visuality (Seattle, WA: Bay Press, 1992), 3-23.

Juris, Jeffrey. 'Practicing Militant Ethnography with the Movement for Global Resistance (MRG) in Barcelona', in Stevphen Shukaitis and David Graeber (eds). Constituent Imagination: Militant Investigation, Collective Theorization (Oakland: AK Press), 164-176.

Kelty, Christopher. Two-Bits: The Cultural Significance of Free Software (Durham: Duke University Press, 2008).

Kerr, Dana. 'Wikileaks Endures a Lengthy DDoS Attack', CNet (2012) http://www.cnet.com/ uk/news/wikileaks-endures-a-lengthy-ddos-attack/

Lazzarato, Maurizio. 'Machines to Crystallize Time: Bergson', Theory, Culture \& Society 24.6 (2007): 93-122.

Lessig, Lawrence. Free Culture (New York: Penguin, 2004).

Lindgren, Simon and Jessica Linde. 'The Subpolitics of Online Piracy: A Swedish Case Study', Convergence: The International Journal of Research into New Media Technologies 18.26 (2012): 143-164.

Manovich, Lev.* The Language of New Media* (Cambridge, Mass: MIT Press, 2001).

McLuhan, Marshall. Understanding Media (New York: McGraw-Hill, 1964).

McKelvey, Fenwick. 'We Like Copies, Just Don't Let the Other Fool You: The Paradox of the Pirate Bay', Television and New Media (2014). 
Microsoft. 'Benefits of Database Mirroring' (2014), http://technet.microsoft.com/en-us/library/ms189852.aspx\#Benefits

Milan Stefania. 'WikiLeaks, Anonymous, and the Exercise of Individuality: Protesting in the Cloud', In Benedetta Brevini, Arne Hintz, and Patrick McCurdy (eds). Beyond Wikileaks. Implications for the Future of Communications, Journalism and Society (London: Palgrave Macmillan, 2013), 191-208.

Mouffe, Chantel. On the Political (New York, NY: Routledge, 2005).

Navas, Eduardo.'Regressive and reflexive mashups in Sampling Culture', Remix Theory (2010) http://remixtheory.net/?p=444

Nielsen, Rasmus K. 'The Labors of Internet-assisted Activism: Overcommunication, Miscommunication, and Communicative Overload', Journal of Information Technology \& Politics 6.3-4 (2009): 267-280.

Ong, Walter J. Interfaces of the Word: Studies in the Evolution of Consciousness and Culture (Ithaca, N.Y.: Cornell University Press, 1977).

Parikka, Jussi. 'Copy', in Matthew Fuller (ed.). Software Studies: A Lexicon (Cambridge, Mass.: MIT Press, 2008), 70-78.

Postigo, Hector. The Digital Rights Movement: The Role of Technology in Subverting Digital Copyright (Cambridge, Mass.: MIT Press, 2012).

Sauter, Molly “LOIC Will Tear Us Apart:" The Impact of Tool Design and Media Portrayals in the Success of Activist DDOS Attacks', American Behaviorial Scientist 57.7 (2013): 9831007.

Scott, James. Seeing Like a State: How Certain Schemes to Improve the Human Condition Have Failed (New Haven: Yale University Press, 1999).

Sifry, Micah. Wikileaks in the Age of Transparency (New York: OR Press, 2011).

Stoehrel, Rodrimigo Ferrada and Simon Lindgren. 'For the Lulz: Anonymous, Aesthetics, and Affect', trip/eC 12.1 (2014) http://www.triple-c.at/index.php/tripleC/article/view/503

Taussig, Michael. Mimesis and Alterity (New York: Routledge, 1993).

Thacker, Eugene. 'Networks, Swarms, Multitudes. Part One', Ctheory (2004), http://www. ctheory.net/articles.aspx?id=422.

Tsing, Anna Lowenhaupt. Friction: An Ethnography of Global Connection (Princeton N.J.: Princeton Press, 2005).

Wiedemann, Carolin. 'Between Swarm, network, and multitude: Anonymous and the infrastructure of the common', Distinktion: Scandinavian Journal of Social Theory, (2014) http:// www.tandfonline.com/doi/full/10.1080/1600910X.2014.895768 
Wikileaks.info. 'Mirrors' (nd), http://wikileaks.info/

Wikileaks.org. (nd), https://wikileaks.org/Mirrors.html

Williams, Linda. 'Film Bodies: Gender, Genre, and Excess', Film Quarterly 44.4 (Summer 1991): 2-13.

The LOCKSS System has the permission to collect, preserve and serve this open access

Archival Unit

This Isuue of the Fibreculture Journal by The Fibreculture Journal Incorporated is licensed under a Creative Commons Attribution 4.0 International License.

\section{OPEN HUMANITIES PRESS}

The Fibreculture Journal is published by The Fibreculture Journal Incorporated in partnership with Open Humanities Press. 